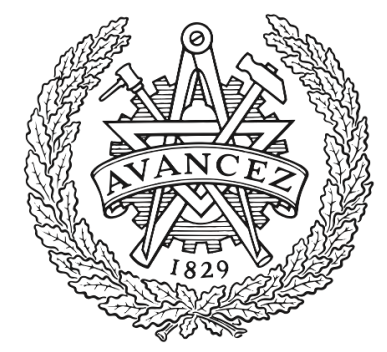

CHALMERS

UNIVERSITY OF TECHNOLOGY

\title{
The Spectral Energy Distribution of the Hyperluminous, Hot Dust-obscured Galaxy W2246-0526
}

Downloaded from: https://research.chalmers.se, 2023-04-26 05:50 UTC

Citation for the original published paper (version of record):

Fan, L., Gao, Y., Knudsen, K. et al (2018). The Spectral Energy Distribution of the Hyperluminous, Hot Dust-obscured Galaxy W2246-0526. Astrophysical Journal, 854(2).

http://dx.doi.org/10.3847/1538-4357/aaaaae

N.B. When citing this work, cite the original published paper. 


\title{
The Spectral Energy Distribution of the Hyperluminous, Hot Dust-obscured Galaxy W2246-0526
}

\author{
Lulu Fan ${ }^{1}$ (10), Ying Gao ${ }^{1}$, Kirsten K. Knudsen ${ }^{2}$ (i), and Xinwen $\mathrm{Shu}^{3}$ (10) \\ ${ }^{1}$ Shandong Provincial Key Lab of Optical Astronomy and Solar-Terrestrial Environment, Institute of Space Science, \\ Shandong University, Weihai, 264209, People's Republic of China; 1lfan@sdu.edu.cn \\ ${ }^{2}$ Department of Space, Earth and Environment, Chalmers University of Technology, Onsala Space Observatory, SE-439 92 Onsala, Sweden \\ ${ }_{3}^{3}$ Department of Physics, Anhui Normal University, Wuhu, Anhui, 241000, People's Republic of China \\ Received 2017 October 12; revised 2018 January 23; accepted 2018 January 23; published 2018 February 21
}

\begin{abstract}
Hot dust-obscured galaxies (Hot DOGs) are a luminous, dust-obscured population recently discovered in the WISE All-Sky survey. Multiwavelength follow-up observations suggest that they are mainly powered by accreting supermassive black holes (SMBHs), lying in dense environments, and being in the transition phase between extreme starburst and UV-bright quasars. Therefore, they are good candidates for studying the interplay between SMBHs, star formation, and environment. W2246-0526 (hereafter, W2246), a Hot DOG at $z \sim 4.6$, has been taken as the most luminous galaxy known in the universe. Revealed by the multiwavelength images, the previous Herschel SPIRE photometry of W2246 is contaminated by a foreground galaxy (W2246f), resulting in an overestimation of its total IR luminosity by a factor of about two. We perform the rest-frame UV/optical-to-far-IR spectral energy distribution (SED) analysis with SED3FIT and re-estimate its physical properties. The derived stellar mass $M_{\star}=4.3 \times 10^{11} M_{\odot}$ makes it among the most massive galaxies with spectroscopic redshift $z>4.5$. Its structure is extremely compact and requires an effective mechanism to puff-up. Most of $(>95 \%)$ its IR luminosity is from AGN torus emission, revealing the rapid growth of the central SMBH. We also predict that W2246 may have a significant molecular gas reservoir based on the dust mass estimation.
\end{abstract}

Key words: galaxies: active - galaxies: high-redshift - galaxies: individual (W2246-0526) - infrared: galaxies submillimeter: galaxies

\section{Introduction}

One of the primary science objectives for the Wide-field Infrared Survey Explorer (WISE; Wright et al. 2010) all-sky survey is to identify the most luminous ultraluminous infrared galaxies (ULIRGs) in the universe. With a so-called WIW2dropout color-selected method (Eisenhardt et al. 2012; Wu et al. 2012), a new population of luminous, dust-obscured galaxies (designated as Hot, Dust-Obscured Galaxies, for short Hot DOGs, by $\mathrm{Wu}$ et al. 2012) has been successfully discovered. Several works have suggested that Hot DOGs, mainly powered by accreting supermassive black holes (SMBHs), may represent a key transition phase during the evolution of massive galaxies, linking starbursts and luminous unobscured quasars (Wu et al. 2012; Bridge et al. 2013; DíazSantos et al. 2016; Fan et al. 2016a, 2016b; Wu et al. 2018).

Among those Hot DOGs with spectroscopic redshift and farinfrared photometry, W2246-0526 (hereafter, W2246) is the most distant one at redshift $z_{\mathrm{opt}}=4.593$ derived from UV/optical emission lines (Wu et al. 2012; Tsai et al. 2015). With ALMA [C II] observations of W2246, Díaz-Santos et al. (2016) measured its redshift at $z_{[\mathrm{C} \mathrm{II}}=4.601$, which shows the [C II] line having a significant redshift compared with the UV/optical emission lines. The previous works used multiwavelength fit to its SED and obtained its total IR luminosity $L_{\mathrm{IR}}=2.2-3.4 \times 10^{14} L_{\odot}$ (Tsai et al. 2015; Fan et al. 2016b). Given the corresponding bolometric luminosity $L_{\text {bol }}=3.5-4.8 \times 10^{14} L_{\odot}$, W2246 had been taken as the most luminous galaxy known in the universe (Díaz-Santos et al. 2016)

Recently, we noted that the IR luminosity of W2246 was likely overestimated due to the contamination of a foreground galaxy to Herschel SPIRE photometry. In Figure 1, we show the multiwavelength images of W2246 and the nearby foreground galaxy, W2246f, which is about $16^{\prime \prime}$ away to the northeast of W2246. Due to the poor resolution of Herschel SPIRE 250, 350, and $500 \mu \mathrm{m}$ bands, it is clear that Herschel SPIRE photometry of W2246 is significantly affected by the contamination of W2246f. We re-measure the Herschel SPIRE flux with point-spread function (PSF) fitting. With the updated flux estimations, we fit the rest-frame UV/optical-to-far-IR SED of W2246 with SED3FIT code (Berta et al. 2013) and obtain the key physical properties. Throughout this work, we assume a standard, flat $\Lambda \mathrm{CDM}$ cosmology (see Komatsu et al. 2011), with $H_{0}=$ $70 \mathrm{~km} \mathrm{~s}^{-1}, \Omega_{M}=0.3$, and $\Omega_{\Lambda}=0.7$.

\section{Data}

In order to construct the rest-frame UV/optical-to-far-IR SEDs of W2246 and W2246f, we compile the available multiwavelength data in the literature. In Figure 1, we show the multiwavelength images centering on W2246, including five SDSS bands $(u, g, r, i$, and $z), H S T$ H-band, four WISE bands (W1, W2, W3, and W4), two Herschel PACS bands (70 and $160 \mu \mathrm{m}$, Poglitsch et al. 2010) and three SPIRE bands (250, 350 , and $500 \mu \mathrm{m}$, Griffin et al. 2010). Two red crosses mark the positions of W2246 $\left(22^{\mathrm{h}} 46^{\mathrm{m}} 07^{\mathrm{s}} .5,-05^{\mathrm{d}} 26^{\mathrm{m}} 35^{\mathrm{s}} .3\right)$ and W2246f $\left(22^{\mathrm{h}} 46^{\mathrm{m}} 08^{\mathrm{s}} .3,-05^{\mathrm{d}} 26^{\mathrm{m}} 24^{\mathrm{s}} .5\right)$ based on the HST H-band image.

Five-SDSS-band photometry and photometric redshift of W2246f have been retrieved from Sloan Digital Sky Survey SkyServer. ${ }^{4}$ The photometric redshift of W2246f $\left(z_{\mathrm{ph}}=0.047\right)$ suggests that it is a foreground galaxy. Instead of retrieving the

\footnotetext{
http://skyserver.sdss.org/
} 


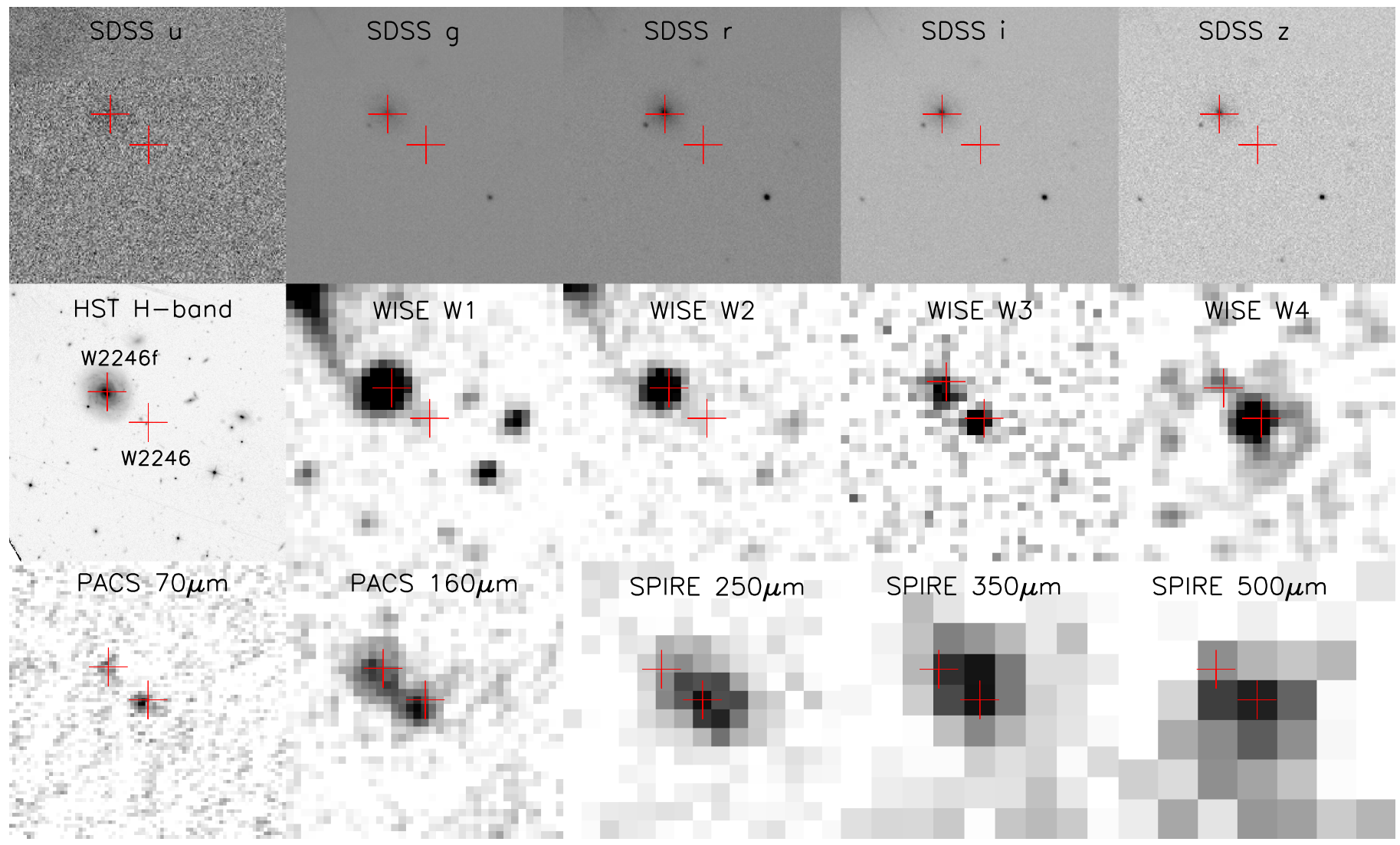

Figure 1. Multiwavelength images of W2246 and its nearby foreground galaxy, W2246f. Top panels: SDSS $u$-, $g$-, $r$-, $i$-, and $z$-band images. Middle panels: $H S T$ H-band, WISE W1,W2,W3, and W4 maps, taken from unWISE images (Lang 2014). Bottom panels, from left to right: Herschel maps in PACS 70 and $160 \mu$ m, SPIRE 250, 350, and $500 \mu \mathrm{m}$, respectively. These maps are $1.5 \times 1.5$ square arcmin in size and centered on W2246. Two crosses mark the positions of W2246 and W2246f based on HST H-band image.
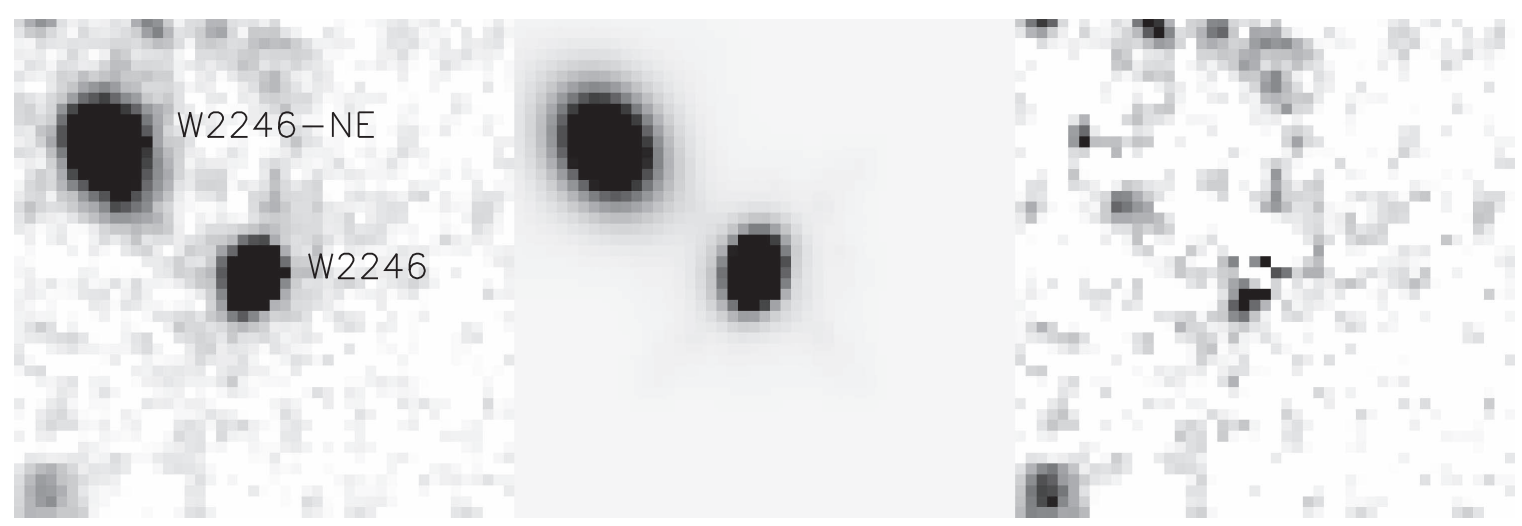

Figure 2. Zoom-in HST H-band image $\left(6^{\prime \prime} \times 6^{\prime \prime}\right)$, including W2246 in the center and its northeast companion (W2246-NE), is presented in the left panel. We use the GALFIT package to fit the surface brightness profiles of W2246 and W2246-NE, simultaneously. For W2246, we adopt a Sérsic + PSF model to represent the host galaxy and AGN component, respectively. For W2246-NE, we only assume a Sérsic model. The model and residual images of W2246 and W2246-NE are shown in the middle and right panels, respectively.

WISE W1, W2, W3, and W4 photometry from the WISE ALLWISE Data Release (Cutri et al. 2013), we do the aperture photometry of both W2246 and W2246f based on the unblurred coadded WISE images ${ }^{5}$ (unWISE, Lang 2014). The photometry errors have been estimated based on the inverse variance images.

W2246f has the 2MASS photometry. We retrieve its near-IR $J$, $H$, and $K_{S}$ flux densities from NED. ${ }^{6}$ In Figure 2, we show a

\footnotetext{
https://unwise.me

6 https://ned.ipac.caltech.edu
}

zoom-in $6^{\prime \prime} \times 6^{\prime \prime} \quad H S T$ H-band image. High-resolution HST H-band image reveals two components: W2246 and its northeast companion (W2246-NE: $22^{\mathrm{h}} 46^{\mathrm{m}} 07^{\mathrm{s}} .6,-05 \mathrm{~d} 26 \mathrm{~m} 33.7 \mathrm{~s}$ ). We derive their photometry by using the GALFIT package (Peng et al. 2002, 2010) to fit the surface brightness profiles of W2246 and W2246NE, simultaneously. For W2246, we adopt a Sérsic + PSF model to represent the host galaxy and AGN component, respectively. For W2246-NE, we only assume a Sérsic model. The model PSF of HST H-band has been constructed by using the TinyTim package (Krist et al. 2011). The Sérsic index $n$ has been set as a free parameter. The best-fit models suggest that both W2246 and 
Table 1

Photometry of W2246 and W2246f

\begin{tabular}{|c|c|c|c|}
\hline \multirow{2}{*}{ Band } & \multirow[t]{2}{*}{$\begin{array}{l}\text { PSF FWHM } \\
\text { (arcsec) }\end{array}$} & \multicolumn{2}{|c|}{ Flux (mJy) } \\
\hline & & W2246 & W2246f \\
\hline SDSS $u$ & 1.5 & $\cdots$ & $0.090 \pm 0.006$ \\
\hline SDSS $g$ & 1.4 & $\ldots$ & $0.387 \pm 0.004$ \\
\hline SDSS $r$ & 1.3 & $<3.9$ (a) & $0.752 \pm 0.007$ \\
\hline SDSS $i$ & 1.2 & $\ldots$ & $1.05 \pm 0.01$ \\
\hline $\operatorname{SDSS} z$ & 1.2 & $\ldots$ & $1.28 \pm 0.03$ \\
\hline 2MASS $J$ & 3.0 & $\ldots$ & $1.60 \pm 0.20$ \\
\hline 2MASS $H$ & 3.0 & $0.0052 \pm 0.0002(\mathrm{~b})$ & $1.55 \pm 0.29$ \\
\hline 2MASS $K_{S}$ & 3.0 & $0.0088 \pm 0.0028(\mathrm{c})$ & $1.43 \pm 0.40$ \\
\hline WISE W1 & 6.1 & $0.031 \pm 0.007$ & $1.16 \pm 0.01$ \\
\hline WISE W2 & 6.4 & $0.034 \pm 0.007$ & $0.70 \pm 0.02$ \\
\hline WISE W3 & 6.5 & $1.2 \pm 0.2$ & $2.52 \pm 0.18$ \\
\hline WISE W4 & 12.0 & $13.0 \pm 1.6$ & $3.17 \pm 0.76$ \\
\hline PACS $70 \mu \mathrm{m}$ & 6.0 & $25.0 \pm 1.3$ & $12.3 \pm 1.2$ \\
\hline PACS $160 \mu \mathrm{m}$ & 11.0 & $56.1 \pm 2.1$ & $65.8 \pm 2.2$ \\
\hline $\begin{array}{l}\text { SPIRE } \\
\qquad 250 \mu \mathrm{m}\end{array}$ & 18.0 & $75.4 \pm 8.3$ & $70.4 \pm 8.3$ \\
\hline $\begin{array}{l}\text { SPIRE } \\
\qquad 350 \mu \mathrm{m}\end{array}$ & 25.0 & $66.0 \pm 8.2$ & $29.1 \pm 8.1$ \\
\hline $\begin{array}{l}\text { SPIRE } \\
\qquad 500 \mu \mathrm{m}\end{array}$ & 38.0 & $57.0 \pm 13.0$ & $<26.0$ \\
\hline $\begin{array}{l}\text { ALMA } \\
\qquad 880 \mu \mathrm{m}\end{array}$ & 0.4 & $7.4 \pm 0.6(\mathrm{~d})$ & $\cdots$ \\
\hline
\end{tabular}

Note. (a) Tsai et al. (2015), (b) HST F160W filter with a PSF FWHM of 0.18", (c) Hale 200-inch WIRC $K_{S}$ (Assef et al. 2015), (d) Díaz-Santos et al. (2016).

W2246-NE have disk-like structures, with $n=0.7$ and 0.8 , respectively. The model and residual images of W2246 and W2246-NE are shown in Figure 2. The remaining flux on the residual image contributes less than 3\% of the total flux of W2246. The pattern of the residual image of W2246 suggests that the system is possibly not yet relaxed after a recent merger. The flux densities of the host galaxy of W2246, its AGN component, and $\mathrm{W} 2246-\mathrm{NE}$ are $5.2 \pm 0.2 \mu \mathrm{Jy}, 0.9 \pm 0.2 \mu \mathrm{Jy}$, and $6.9 \pm 0.1 \mu \mathrm{Jy}$, respectively. The $K_{S}$ band photometry of W2246 is taken from Assef et al. (2015), which was observed by Hale P200 WIRC.

Herschel flux densities and their associated uncertainties have been derived from PSF fitting using WISE $12 \mu \mathrm{m}$ sources as prior positions. During our fittings, the $12 \mu \mathrm{m}$ prior positions are fixed, as for the longest passbands of SPIRE (i.e., $350 \mu \mathrm{m}$ and $500 \mu \mathrm{m}$ ), the increasingly large PSFs make the source of interest and its close neighbor strongly blended. For PACS $70 \mu \mathrm{m}$ and $160 \mu \mathrm{m}$ observations that have better spatial resolution, we find little difference in flux measurements if the prior positions are allowed to vary. Dust continuum emission of W2246 at $\sim 880 \mu \mathrm{m}$ has been resolved by ALMA observations and its flux density is $7.4 \pm 0.6 \mathrm{mJy}$ (Díaz-Santos et al. 2016). We summarize the photometry of W2246 and W2246f in Table 1.

\section{SED Fitting}

For Hot DOGs, Assef et al. (2015) modeled their rest-frame optical through mid-IR SEDs following the approach applied in Eisenhardt et al. (2012). Each SED had been modeled as a combination of the host galaxy template and one AGN SED template. Their result showed that the median value of AGN obscuration for Hot DOGs is $E(B-V)=6.0$. The stellar component dominated the optical-to-near-IR SED, while the AGN component dominated the mid-IR band. In our previous work (Fan et al. 2016b), we modeled the IR SEDs of 22 Hot DOGs using two main components: dust emission from star formation and AGN torus emission. Our result showed that the two-component model can fit the observed IR SEDs of Hot DOGs well and AGN torus emission dominated the IR energy output.

We construct the rest-frame UV/optical-to-far-IR SED of W2246 using the data set described in Section 2. At least three components, including stellar emission, dust emission from star formation, and AGN torus emission, can contribute to the UV/optical-to-far-IR SED of W2246. Here, we use the three-component SED-fitting code SED3FIT by Berta et al. (2013), ${ }^{7}$ which implements the Multiwavelength Analysis of Galaxy Physical Properties code MAGPHYS (da Cunha et al. 2008) ${ }^{8}$ with an additional AGN torus component from the library of Fritz et al. (2006) and Feltre et al. (2012), to model the observed SED of W2246. We adopt Bruzual \& Charlot (2003) optical/near-IR stellar library and Chabrier (2003) initial mass function (IMF). However, we cannot rule out the possibility that a small fraction of the rest-frame $\mathrm{UV} /$ optical emission of the luminous AGN is leaked out of the high-obscuration region. As mentioned by Assef et al. (2016), a fraction of the AGN light can be possibly scattered off into our line of sight. We will discuss this possibility in Section 4.

We use the same three-component SED3FIT code to fit the rest-frame UV/optical-to-far-IR SED of W2246f. In Figure 3, the solid line shows the best-fit result of W2246f using SED3FIT. The dashed and dashed-dotted lines represent the attenuated stellar emission and dust emission from star formation, respectively. The dotted line shows the AGN torus emission.

\section{Results and Discussion}

In Figure 4, we present the best-fit model SED (solid line) of W2246 with SED3FIT. The three-component model provides a rather good description of the rest-frame UV/optical-to-far-IR SED with $\chi^{2}=1.53$. The deviation at $500 \mu \mathrm{m}$ band is possibly due to the large uncertainty of PSF fitting photometry. Derived physical properties of W2246 have been listed in Table 2, including the total IR luminosity $\left(L_{\mathrm{IR}}^{\text {tot }}\right)$, the IR luminosity contributed by AGN torus $\left(L_{\mathrm{IR}}^{\text {torus }}\right)$, the IR luminosity related to star formation $\left(L_{\mathrm{IR}}^{\mathrm{SF}}\right)$, the far-IR luminosity related to star formation $\left(L_{\mathrm{FIR}}^{\mathrm{SF}}\right)$, star formation rate (SFR), stellar masses $\left(M_{\star}\right)$, and dust mass $\left(M_{\text {dust }}\right)$. We plot the likelihood distributions of $M_{\star}, \mathrm{SFR}, L_{\mathrm{IR}}^{\mathrm{SF}}$ and $M_{\text {dust }}$ in Figure 5 .

The total IR luminosity is lower than the previous estimations (Jones et al. 2014; Fan et al. 2016b) by a factor of about two due to taking out the contamination of the foreground galaxy W2246f to SPIRE photometry. AGN torus emission, contributing over $95 \%$ of the total IR luminosity, not only dominates in the mid-IR wavelength range, but also has a significant contribution up to the rest-frame $100 \mu \mathrm{m}$. At the rest-frame wavelength range longer than $100 \mu \mathrm{m}$, dust emission related to star formation starts to dominate. After deducting the AGN contribution, the far-IR luminosity related to star formation is only $2.1 \times 10^{12} L_{\odot}$, which is lower than that

\footnotetext{
http://cosmos.astro.caltech.edu/page/other-tools

8 http://www.iap.fr/magphys/
} 


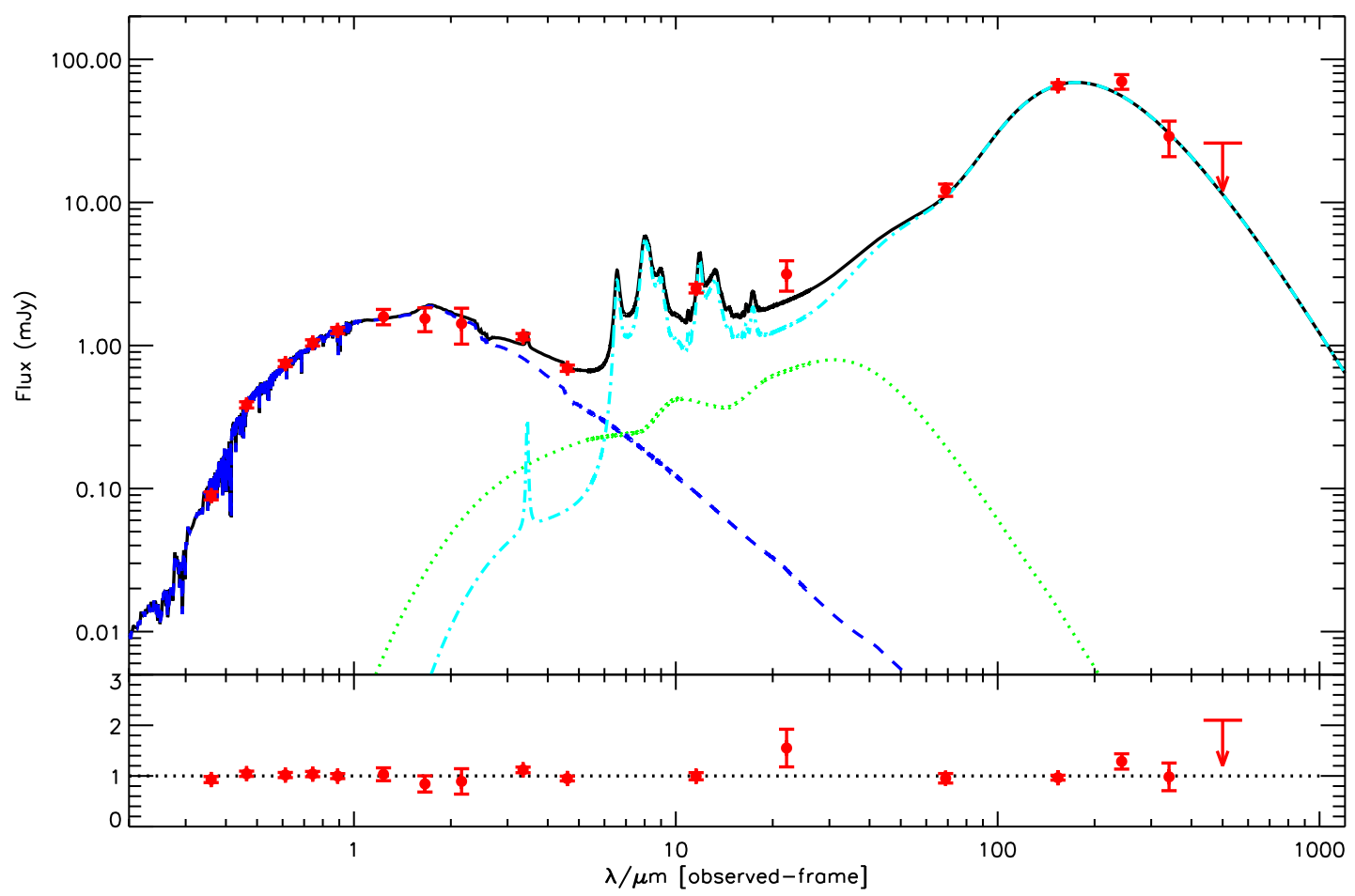

Figure 3. Best-fit model SED (solid line) of W2246f with SED3FIT. The observed data are listed in Table 1 and plotted here with red circles. The blue dashed line represents the attenuated stellar emission, and the cyan dashed-dotted line represents dust emission from star formation. The green dotted line shows the AGN torus dust emission. The bottom panel shows the ratio of the observed flux to model prediction.

used in Díaz-Santos et al. (2016) by one order of magnitude. Adopting the total $[\mathrm{C} \mathrm{II}]$ luminosity of W2246 $L_{[\mathrm{C} \mathrm{II}]}=$ $6.1 \times 10^{9} L_{\odot}$, our estimation of the far-IR luminosity results in the [C II]-to-far-IR emission ratio ([C II]/FIR) of $2.9 \times$ $10^{-3}$, which is similar to some high-redshift ULIRGs (De Looze et al. 2014) and quasars at $z>4$ (Willott et al. 2013, 2015; Venemans et al. 2016). The previously reported [C II]/FIR deficit of W2246 (e.g., Díaz-Santos et al. 2016) and other high-redshift quasars (Wang et al. 2013) may be at least in part due to AGN contamination of the far-IR emission.

UV/Optical SED is dominated by stellar emission. The derived stellar mass of W2246 is $4.3 \times 10^{11} \mathrm{M}_{\odot}$, which is among the most massive galaxies with spectroscopic redshift $z>4.5$ (Caputi et al. 2015). In order to consider the effects of different IMF, metallicity, and star formation history (SFH) on the stellar mass estimation, we utilize FAST (Kriek et al. 2009) to fit the observed $H, K_{S}, W 1$, and $W 2$ bands. The derived stellar mass can change by a factor of up to $0.3 \mathrm{dex}$, adopting the different combination of IMF, metallicity, and SFH. It is possible that the stellar mass can be overestimated by the contamination of AGN emission (for instance, the scattered AGN emission) to optical/near-IR bands. We consider the possible contribution of AGN emission by doing the structural decomposition using the GALFIT package (Peng et al. 2002, 2010). The high spatial resolution HST H-band image of W2246 has been decomposed with a Sérsic + PSF model. The decomposed PSF component has the flux $0.9 \pm 0.2 \mu \mathrm{Jy}$, which is about six times weaker than the Sérsic component. We assume that the PSF component comes from the scattered AGN emission as suggested by Assef et al. (2016). In Figure 4, an attenuated Type 1 QSO SED (Richards et al. 2006) has been plotted to present this scattered AGN emission. The dust attenuation of host galaxy has been set to $A_{V}=0.5$, which is determined by the SED3FIT result. The scattered AGN emission is lower than stellar emission by over one order of magnitude in the optical and near-IR bands. Thus, the AGN contamination has a negligible effect on the stellar mass measurement of W2246.

The structural parameters, Sérsic index $n=0.7$ and effective radius $R_{e}=1.3 \mathrm{Kpc}$, of $\mathrm{W} 2246$ have been derived based on the Sérsic + PSF model, making it a disk-like, extremely compact galaxy at such a high redshift. Díaz-Santos et al. (2016) showed even more compact structures of [C II] emission line and dust continuum than UV continuum. Such a compact galaxy is expected to evolve into a red nugget at $z \sim 2-3$ and experience a dramatic structural evolution. In order to catch up the local masssize relation of massive early-type galaxies (Shen et al. 2003), W2246 would require an increase in its present size of a factor of $\sim 7$. AGN feedback, which is taking action to blow out the ISM in W2246 (Díaz-Santos et al. 2016), will possibly play an important role in such a dramatic size increase, as suggested by our previous model (Fan et al. 2008, 2010). Another mechanism, like a dry minor merger (e.g., Naab et al. 2009), may also contribute the observed size evolution during the late evolutionary stage. Recently, a new scenario to explain the evolution of extremely compact galaxies at high redshift supposes that they survive as the compact cores (bulge components) embedded in present-day massive galaxies (Graham et al. 2015; de la Rosa et al. 2016).

The derived SFR of $\mathrm{W} 2246$ is $480 M_{\odot} \mathrm{yr}^{-1}$, which is comparable to some starburst galaxies. However, considering its high redshift and large stellar mass, W2246 still lies below the the star-forming galaxy (SFG) main sequence (MS), which suggests $\mathrm{SFR} \sim 1100 M_{\odot} \mathrm{yr}^{-1}$ for an MS galaxy with $4.3 \times 10^{11} M_{\odot}$ stellar mass and at the age of the universe $t \sim 1.2 \mathrm{Gyr}$ (Speagle et al. 2014). This result suggests that 


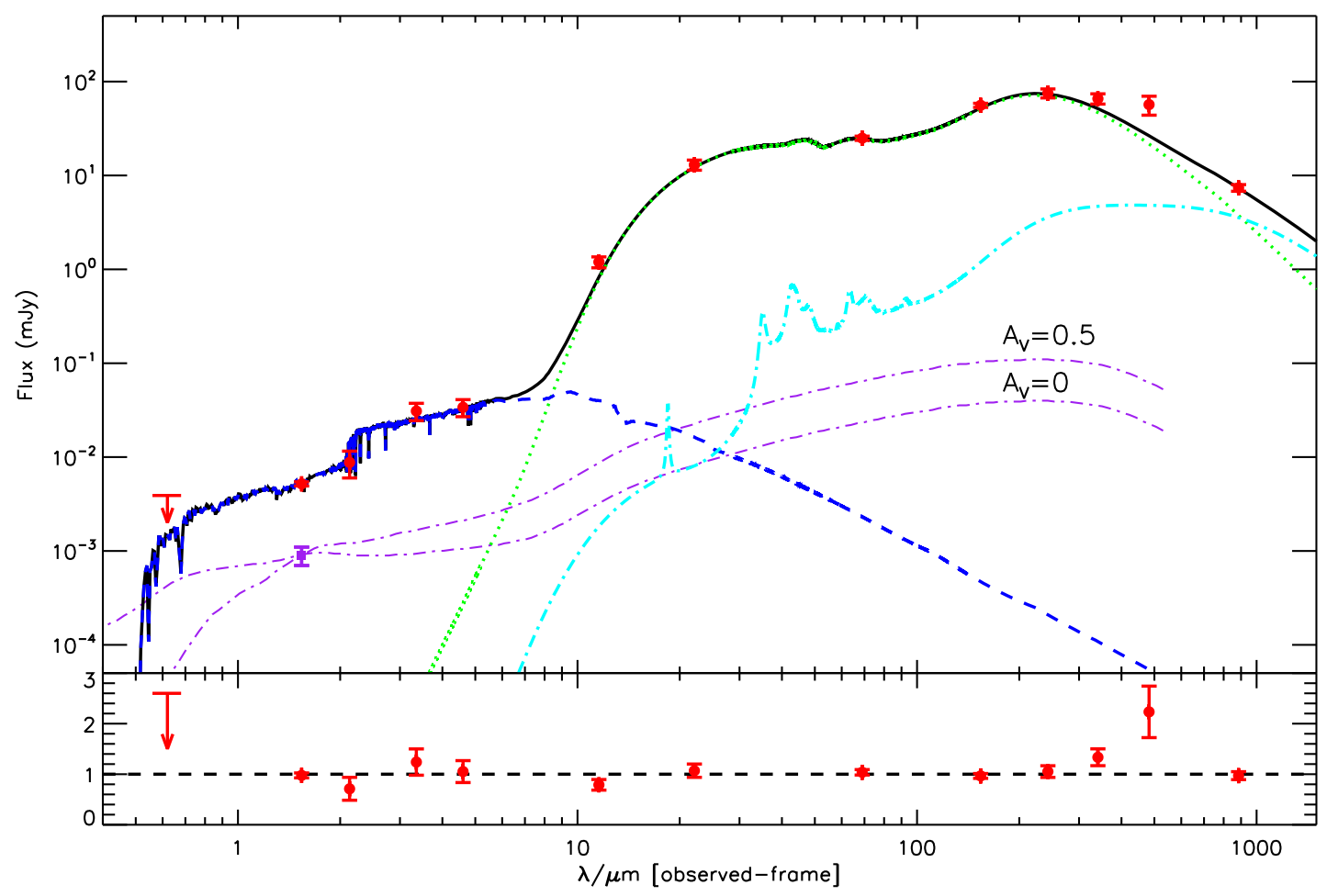

Figure 4. Best-fit model SED (solid line) of W2246 with SED3FIT. The red circles are the observed data points. The blue dashed and cyan dashed-dotted lines show the attenuated stellar emission and dust emission from star formation, respectively. The green dotted line is the contribution of AGN torus emission. The flux contributed by AGN emission in H-band, which is shown as the purple filled square, has been estimated based on the structural decomposition using GALFIT. We plot the mean SEDs of Type 1 QSOs (Richards et al. 2006), unattenuated and attenuated by dust assuming a SMC-like extinction law (Prevot et al. 1984) with $A_{V}=0.5$. The unattenuated and attenuated Type 1 QSO SEDs (purple dashed-dotted lines) have been normalized to the flux of AGN emission in H-band. The bottom panel shows the ratio of the observed flux to model prediction.

Table 2

Physical Properties of W2246 and W2246f

\begin{tabular}{lcc}
\hline \hline & W2246 & W2246f \\
\hline$L_{\mathrm{IR}[8-1000 \mu \mathrm{m}]}^{\text {tot }}\left[L_{\odot}\right]$ & $1.2 \times 10^{14}$ & $3.2 \times 10^{9}$ \\
$L_{\mathrm{IR}[8-1000 \mu \mathrm{m}]}^{\text {torus }}\left[L_{\odot}\right]$ & $1.1 \times 10^{14}$ & $2.1 \times 10^{8}$ \\
$L_{\mathrm{IR}[8-1000 \mu \mathrm{m}]}^{\mathrm{SF}}\left[L_{\odot}\right]$ & $4.5 \times 10^{12}$ & $3.0 \times 10^{9}$ \\
$L_{\mathrm{FIR}[42-122 \mu \mathrm{m}]}^{\mathrm{SF}}\left[L_{\odot}\right]$ & $2.1 \times 10^{12}$ & $1.0 \times 10^{9}$ \\
$S F R\left[M_{\odot} \mathrm{yr}-1\right]$ & $480_{-217}^{+70}$ & $0.09_{-0.02}^{+0.07}$ \\
$M_{\star}\left[M_{\odot}\right]$ & $4.3_{-1.5}^{+2.3} \times 10^{11}$ & $1.1_{-0.2}^{+0.5} \times 10^{10}$ \\
$M_{\text {dust }}\left[M_{\odot}\right]$ & $9.1_{-4.5}^{+0.4} \times 10^{8}$ & $1.4_{-0.4}^{+1.6} \times 10^{7}$ \\
\hline
\end{tabular}

W2246 may be experiencing the declining and quenching of star formation.

By integrating the best-fit model SED of W2246, we derive its bolometric luminosity $L_{\text {bol }}=1.7 \times 10^{14} L_{\odot}$. Assuming that the SMBH in the center of W2246 accretes at the Eddington ratio $\eta=1$ (Wu et al. 2018), the estimated black hole mass is $5.1 \times 10^{9} M_{\odot}$. The corresponding black hole-bulge mass ratio $\left(M_{\mathrm{BH}} / M_{\text {bulge }}\right)$ is 0.012 , which is about 2.4 times higher than the present-day value, suggesting that the SMBH accumulates most of its mass before the formation of the stellar bulge. Both the $M_{\mathrm{BH}}$ and $M_{\mathrm{BH}} / M_{\text {bulge }}$ ratio of W2246 are in agreement with those of many other high-redshift quasars (e.g., Peng et al. 2006; Wang et al. 2010). The present-day $M_{\mathrm{BH}} / M_{\text {bulge }}$ ratio, which has been recently updated by Kormendy \& Ho (2013), is about 0.0049 at $M_{\text {bulge }}=10^{11} M_{\odot}$ and is 2-4 times larger than previous values ranging from 0.001 to 0.0023 . Considering the large intrinsic scatter $(0.29 \mathrm{dex})$ of the present-day $M_{\mathrm{BH}} / M_{\text {bulge }}$ ratio, only a moderate evolution of the $\mathrm{BH}$ mass ratio of
W2246 is required to reach the present-day $M_{\mathrm{BH}}-M_{\text {bulge }}$ relation. W2246 is expected to evolve toward the most massive galaxy hosting a monster black hole in the local universe.

The derived dust mass of $M_{\text {dust }}=9.1 \times 10^{8} M_{\odot}$ indicates that there is likely a large amount of molecular gas $\left(\sim 10^{11} M_{\odot}\right)$ in W2246. The idea that luminous Hot DOGs may have plenty of molecular gas has been supported by our recent ALMA CO observations of three Hot DOGs (Fan et al. 2017b), which find that all of them have a significant molecular gas reservoir $\left(\sim 10^{10-11} M_{\odot}\right)$. The ongoing ALMA CO observations of W2246 will help measure its molecular gas directly.

\section{Conclusions}

W2246, a WISE-selected, hyperluminous dust-obscured galaxy at $z=4.593$, was taken as the most luminous galaxy known in the universe. However, according to the multiwavelength images (see Figure 1), we noted that the previous Herschel SPIRE photometry of W2246 was contaminated by a foreground galaxy (W2246f), resulting in an overestimation of its total IR luminosity. Based on the new WISE and Herschel SPIRE photometry, we perform a SED analysis on the rest-frame UV/optical-to-far-IR of W2246 with SED3FIT. The derived total IR luminosity is about two times lower than the previous estimations, unseating it as the most luminous Hot DOG.

The results from the new SED-fitting show that W2246 is a very interesting object, as it is in a key transition phase during the evolution of massive galaxies. With the derived stellar mass $M_{\star}=4.3 \times 10^{11} M_{\odot}$, it is among the most massive galaxies with spectroscopic redshift $z>4.5$. Besides the high stellar mass, its structure is extremely compact, which indicates that it 

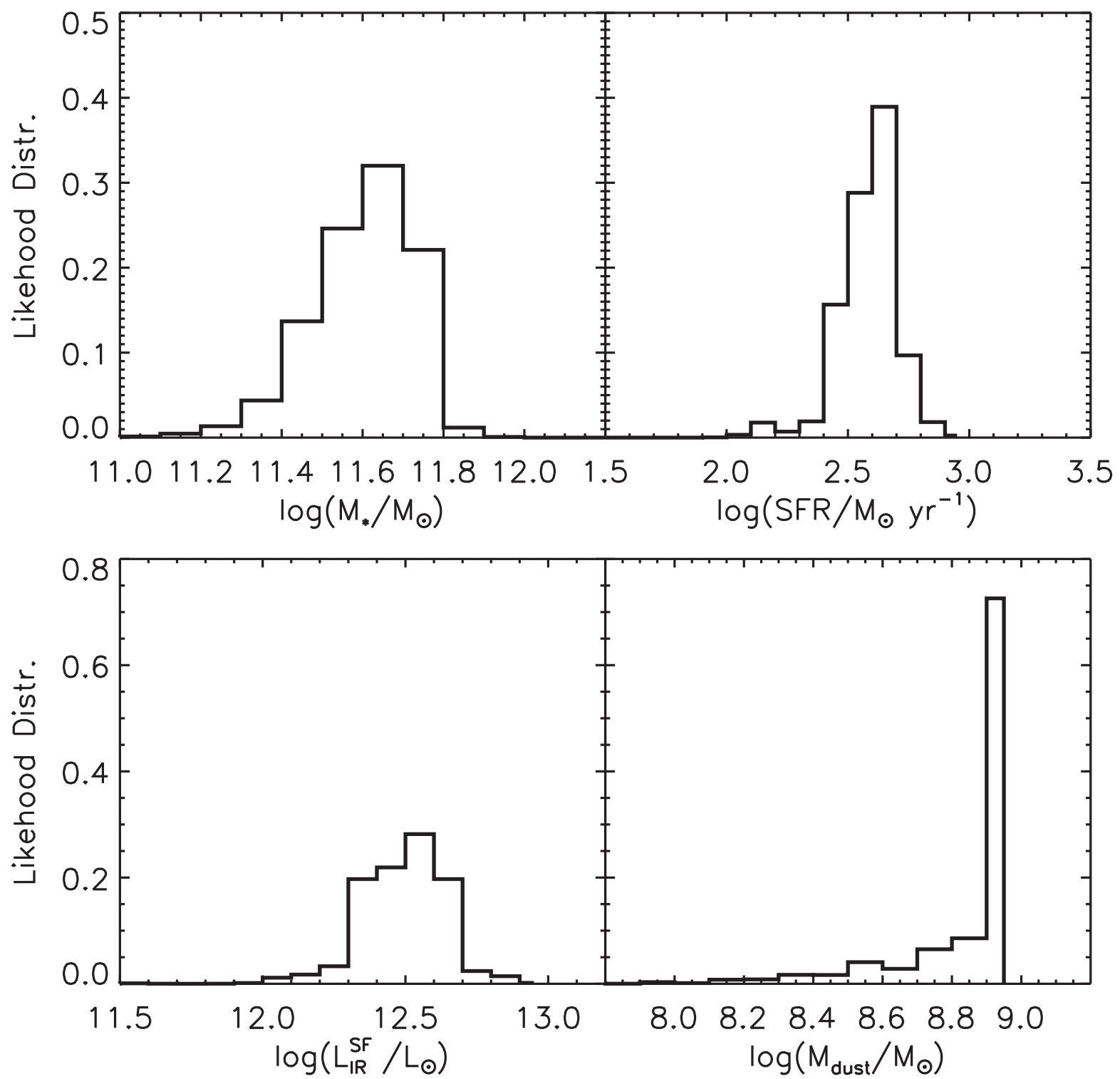

Figure 5. Likelihood distributions of stellar mass $\left(M_{\star}\right)$, star formation rate (SFR), dust luminosity related to star formation $\left(L_{\mathrm{IR}}^{\mathrm{SF}}\right)$, and dust mass $\left(M_{\mathrm{dust}}\right)$ of W2246.

will experience a dramatic size evolution toward low redshift. Most of ( $>95 \%)$ its IR luminosity is from AGN torus emission, revealing the rapid growth of the central SMBH according to the accretion. Although the derived SFR is high $\left(480 M_{\odot} \mathrm{yr}^{-1}\right)$, it still lies below the star-forming galaxy main sequence. Therefore, it has been suggested to be experiencing the declining and quenching of star formation. We also predict that W2246 may have a significant molecular gas reservoir, which can be tested by the ongoing ALMA CO line observations. Both AGN and star formation activities in W2246 may be related to its environment. It is possible that W2246 lies in an overdense environment, which has been suggested by several previous works (e.g., Jones et al. 2014; Assef et al. 2015; Fan et al. 2017a). Our ongoing work with the VLT FORS2 narrow-band imaging will shed insight on the environment of Ly $\alpha$ emitters (LAEs) around it.

The authors would like to thank the anonymous referee for his/her comments and suggestions, which have greatly improved this paper. We thank Dr. Chen Cao and Ms. Berzaf Berhane for their valuable discussions on Herschel photometry and the usage of SED3FIT. L.F. acknowledges the support from National Key Research and Development Program of China (No. 2017YFA0402703). This work is supported by the National Natural Science Foundation of China (NSFC; grant Nos. 11773020, 11433005, and 11573001) and Shandong Provincial Natural Science Foundation, China (ZR2017QA001). K.K. acknowledges the Knut and Alice Wallenberg Foundation for support.

Facilities: WISE, Herschel (PACS, SPIRE), HST, SDSS.

\section{ORCID iDs}

Lulu Fan (iD https://orcid.org/0000-0003-4200-4432

Kirsten K. Knudsen (iD https://orcid.org/0000-0002-7821-8873

Xinwen Shu (D) https://orcid.org/0000-0002-7020-4290

\section{References}

Assef, R. J., Eisenhardt, P. R. M., Stern, D., et al. 2015, ApJ, 804, 27 Assef, R. J., Walton, D. J., Brightman, M., et al. 2016, ApJ, 819, 111 Berta, S., Lutz, D., Santini, P., et al. 2013, A\&A, 551, A100

Bridge, C. R., Blain, A., Borys, C. J. K., et al. 2013, ApJ, 769, 91 
Bruzual, G., \& Charlot, S. 2003, MNRAS, 344, 1000

Caputi, K. I., Ilbert, O., Laigle, C., et al. 2015, ApJ, 810, 73

Chabrier, G. 2003, PASP, 115, 763

Cutri, R. M., et al. 2013, yCat, 2328, 0

da Cunha, E., Charlot, S., \& Elbaz, D. 2008, MNRAS, 388, 1595

de la Rosa, I. G., La Barbera, F., Ferreras, I., et al. 2016, MNRAS, 457, 1916

De Looze, I., Cormier, D., Lebouteiller, V., et al. 2014, A\&A, 568, A62

Díaz-Santos, T., Assef, R. J., Blain, A. W., et al. 2016, ApJL, 816, L6

Eisenhardt, P. R. M., Wu, J., Tsai, C.-W., et al. 2012, ApJ, 755, 173

Fan, L., Han, Y., Fang, G., et al. 2016a, ApJL, 822, L32

Fan, L., Han, Y., Nikutta, R., Drouart, G., \& Knudsen, K. K. 2016b, ApJ, 823, 107

Fan, L., Jones, S. F., Han, Y., \& Knudsen, K. K. 2017a, PASP, 129, 124101

Fan, L., Knudsen, K. K., Fogasy, J., \& Drouart, G. 2017b, arXiv:1711.10615

Fan, L., Lapi, A., Bressan, A., et al. 2010, ApJ, 718, 1460

Fan, L., Lapi, A., De Zotti, G., \& Danese, L. 2008, ApJL, 689, L101

Feltre, A., Hatziminaoglou, E., Fritz, J., \& Franceschini, A. 2012, MNRAS, 426, 120

Fritz, J., Franceschini, A., \& Hatziminaoglou, E. 2006, MNRAS, 366, 767

Graham, A. W., Dullo, B. T., \& Savorgnan, G. A. D. 2015, ApJ, 804, 32

Griffin, M. J., Abergel, A., Abreu, A., et al. 2010, A\&A, 518, L3

Jones, S. F., Blain, A. W., Stern, D., et al. 2014, MNRAS, 443, 146

Komatsu, E., Smith, K. M., Dunkley, J., et al. 2011, ApJS, 192, 18

Kormendy, J., \& Ho, L. C. 2013, ARA\&A, 51, 511
Kriek, M., van Dokkum, P. G., Labbé, I., et al. 2009, ApJ, 700, 221

Krist, J. E., Hook, R. N., \& Stoehr, F. 2011, Proc. SPIE, 8127, 81270J

Lang, D. 2014, AJ, 147, 108

Naab, T., Johansson, P. H., \& Ostriker, J. P. 2009, ApJL, 699, L178

Peng, C. Y., Ho, L. C., Impey, C. D., \& Rix, H.-W. 2002, AJ, 124, 266

Peng, C. Y., Ho, L. C., Impey, C. D., \& Rix, H.-W. 2010, AJ, 139, 2097

Peng, C. Y., Impey, C. D., Rix, H.-W., et al. 2006, ApJ, 649, 616

Poglitsch, A., Waelkens, C., Geis, N., et al. 2010, A\&A, 518, L2

Prevot, M. L., Lequeux, J., Prevot, L., Maurice, E., \& Rocca-Volmerange, B. 1984, A\&A, 132, 389

Richards, G. T., Lacy, M., Storrie-Lombardi, L. J., et al. 2006, ApJS, 166, 470

Shen, S., Mo, H. J., White, S. D. M., et al. 2003, MNRAS, 343, 978

Speagle, J. S., Steinhardt, C. L., Capak, P. L., \& Silverman, J. D. 2014, ApJS, 214,15

Tsai, C.-W., Eisenhardt, P. R. M., Wu, J., et al. 2015, ApJ, 805, 90

Venemans, B. P., Walter, F., Zschaechner, L., et al. 2016, ApJ, 816, 37

Wang, R., Carilli, C. L., Neri, R., et al. 2010, ApJ, 714, 699

Wang, R., Wagg, J., Carilli, C. L., et al. 2013, ApJ, 773, 44

Willott, C. J., Bergeron, J., \& Omont, A. 2015, ApJ, 801, 123

Willott, C. J., Omont, A., \& Bergeron, J. 2013, ApJ, 770, 13

Wright, E. L., Eisenhardt, P. R. M., Mainzer, A. K., et al. 2010, AJ, 140, 1868

Wu, J., Jun, H. D., Assef, R. J., et al. 2018, ApJ, 852, 96

Wu, J., Tsai, C.-W., Sayers, J., et al. 2012, ApJ, 756, 96 